\title{
Aspectos biológicos, morfológicos e comportamentais de Aspidiotus nerii Bouché, 1833 (Hemiptera: Diaspididae)
}

\author{
Biological, morphological and behavioral aspects of Aspidiotus nerii Bouché, 1833 (Hemiptera: Diaspididae)
}

\author{
Kelly Cristina Gonçales Rocha ${ }^{1}$ Ricardo Adaime da Silva² Marcos Doniseti Michelotto $^{3}$ \\ Antonio Carlos Busoli ${ }^{4}$
}

\section{RESUMO}

O presente trabalho teve por objetivo estudar os aspectos biológicos, morfológicos e comportamentais de uma linhagem unipariental de Aspidiotus nerii Bouché, 1833 (Hemiptera: Diaspididae), sob condições controladas $\left(25 \pm 1^{\circ} \mathrm{C}\right.$, $70 \pm 10 \%$ de UR e 12 horas de fotofase), tendo abóboras híbridas "cabotiá" como hospedeiro. Ninfas recém-eclodidas foram transferidas da criação massal para abóboras "cabotiá", devidamente higienizadas, dispostas em câmaras climatizadas.

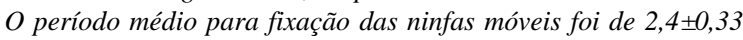
horas. A duração média do $1^{o}$ e $2^{o}$ estádios, após a fixação das

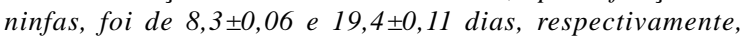

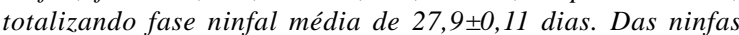
que atingiram a fase adulta, 36 fêmeas foram acompanhadas diariamente até o final do ciclo. A duração média dos períodos

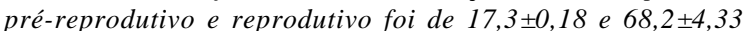
dias, respectivamente. O número total médio de ninfas/fêmea foi 175,5 $\pm 10,29$ e o número médio de ninfas/fêmea/dia foi $2,7 \pm 0,11$. A longevidade média das fêmeas foi $100,5 \pm 4,51$ dias.

Palavras-chave: Cochonilha-de-carapaça, criação massal, biologia.

\section{ABSTRACT}

The objective of this work was to study the biology, morphology and behavior of Aspidiotus nerii Bouché, 1833 (Hemiptera: Diaspididae) under controlled conditions $\left(25 \pm 1^{\circ} \mathrm{C}\right.$, $70 \pm 10 \%$ R.H. and 12 hours of photophase), using "cabotiá" cucumber pumpkins as hosts. Nymphs of the 1st stage obtained of mass rearing (mother culture) were transfered to "cabotiá" pumpkins, properly cleaned and disposed in acclimatized cameras. The medium period for fixation of the nymphs was of $2.4 \pm 0.33$ hours. The 1st and 2nd stages, after the fixation of the nymphs, lasted $8.3 \pm 0.06$ and $19.4 \pm 0.11$ days, respectively, totalizing a nymphal phase of $27.9 \pm 0.11$ days. 36 females were observed daily until the end of the cycle. The pre-reproductive and reproductive periods lasted $17.3 \pm 0.18$ and $68.2 \pm 4.33$ days, respectively. The medium total number of nymphs/female was $175.5 \pm 10.29$ and the medium number of nymphs/female/day was $2.7 \pm 0.11$. The female's longevity was $100.5 \pm 4.51$ days.

Keys words: Oleander scale, mass rearing, biology.

\section{INTRODUÇÃO}

Aspidiotus nerii é uma espécie cosmopolita de cochonilha-de-carapaça, extremamente polífaga e pode se reproduzir de forma sexuada ou assexuada, nesse último caso por partenogênese telítoca (BEARDSLEY JÚNIOR \& GONZALEZ, 1975). Apresenta formato circular, achatado, corpo com coloração amarelada e coberto por uma carapaça de cera, e, como outros diaspidídeos, na fase adulta não apresentam asas, pernas e olhos (ZIMMERMAN, 1948). Segundo NEUENSCHWANDER et al. (1977), a distribuição de $\boldsymbol{A}$. nerii no interior das plantas mostra que a espécie prefere microclima protegido, sendo que as de linhagem unipariental e bipariental apresentam taxas diferentes de desenvolvimento em relação à temperatura (TOMKINS et al., 1997).

${ }^{1}$ Curso de Mestrado em Entomologia Agrícola, Faculdade de Ciências Agrárias e Veterinárias (FCAV), Universidade Estadual Paulista (UNESP), Via de Acesso Prof. Paulo Donato Castellane, s/n, 14884-900, Jaboticabal, São Paulo, Brasil. E-mail: kellycgr@yahoo.com.br.

2Embrapa Amapá, Rodovia JK, km 5, 68903-000, Macapá, Amapá, Brasil. E-mail: adaime@cpafap.embrapa.br. Autor para correspondência.

${ }^{3}$ Doutorando em Entomologia Agrícola, FCAV/UNESP, Jaboticabal, SP, Brasil. E-mail: mdmichelotto@yahoo.com.br.

${ }^{4}$ Departamento de Fitossanidade, FCAV/UNESP, Jaboticabal, SP, Brasil. E-mail: acbusoli@fcav.unesp.br. 
Atualmente, a cochonilha $\boldsymbol{A}$. nerii, apesar de não ser considerada praga, encontra-se disseminada por algumas regiões do Brasil em diversos hospedeiros. SILVA et al. (1968) destacaram a sua presença em várias culturas, sem muita ênfase aos danos causados.

Trabalhos visando ao estudo da bioecologia de insetos em diversos hospedeiros buscam adequar qualidade e baixo custo com aumento da viabilidade dos insetos, fornecendo informações sobre a possibilidade de criação massal desses indivíduos para a utilização em projetos de controle biológico (GARCIA, 1991).

Os diaspidídeos desenvolvem-se facilmente em condições de laboratório, apresentam ciclo rápido e muitas gerações anuais (FLANDERS, 1951; DeBACH \& WHITE, 1960; TASHIRO, 1966; ROSE, 1990). No Brasil, vários pesquisadores desenvolvem estudos com a criação massal de $\boldsymbol{A}$. nerii para sustentar a criação de coccinelídeos predadores, como Pentilia egena Mulsant, 1850 (GUERREIRO, 2000) e Coccidophilus citricola Brèthes, 1905 (SILVA et al., 2003). BUSOLI et al. (2005) criaram com sucesso o predador Cybocephalus sp. (Coleoptera: Nitidulidae) sobre a mesma cochonilha. Tanto os coccinelídeos quanto o nitidulídeo se alimentam da cochonilha e depositam seus ovos sob as carapaças das mesmas. As cochonilhas que têm sido utilizadas são oriundas de uma linhagem uniparental de $\boldsymbol{A}$. nerii, importada do USDA e da Estação Experimental de Lake Alfred, da Universidade da Flórida, Estados Unidos, pelo Prof. Dr. Harold Browning, no ano de 1992. Desde então, vem sendo criada nos laboratórios do Departamento de Fitossanidade da FCAV/Unesp.

Estudos bioecológicos dos insetos utilizados como alimentos alternativos na manutenção de uma criação massal de inimigos naturais, particularmente os predadores, são fundamentais. No caso de $\boldsymbol{A}$. nerii, são poucas as informações existentes quanto à sua biologia, principalmente desta linhagem unipariental.

Este trabalho teve como objetivo estudar os aspectos biológicos, morfológicos e comportamentais de $\boldsymbol{A}$. nerii, em condições de laboratório, tendo como substrato para a sua colonização, abóboras híbridas “cabotiá”, com vistas ao aprimoramento da criação massal de coccinelídeos e outros predadores e parasitoídes.

\section{MATERIAL E MÉTODOS}

O ensaio foi realizado no Laboratório de Bioecologia de Coccinellidae e Diaspididae do Departamento de Fitossanidade, da Faculdade de Ciências Agrárias e Veterinárias da Universidade Estadual Paulista, em Jaboticabal, SP.

Uma linhagem uniparental da cochonilha $\boldsymbol{A}$. nerii, proveniente da Flórida, EUA, foi mantida em criação massal, sobre abóboras híbridas japonesas “cabotiá” (Cucurbita moschata x C. maxima var. tetsukabuto), dispostas em estantes de aço. Os procedimentos utilizados foram semelhantes aos descritos por ROSE (1990), que consistem em dispor abóboras matrizes infestadas pela cochonilha na prateleira superior da estante e, nas prateleiras inferiores, são dispostas as abóboras a serem colonizadas. A colonização ocorre por ninfas de $1^{\circ}$ estádio, móveis, que ao caminharem pela abóbora à procura de um local para sua fixação e alimentação, caem por gravidade sobre as abóboras das prateleiras inferiores, passando a colonizá-las.

Foram utilizadas abóboras com diâmetro médio de $15 \mathrm{~cm}$, casca com leves reentrâncias, sem ferimentos e com pedúnculo de no mínimo $2 \mathrm{~cm}$. As abóboras foram lavadas com água e sabão, com o auxílio de uma esponja, e posteriormente mergulhadas em solução de hipoclorito de sódio $(0,25 \%)$ por um minuto. Após secarem naturalmente, foram dispostas na estante, de acordo com a demanda por cochonilhas.

Para descrever os aspectos morfológicos e comportamentais de A. nerii, ninfas e adultos foram observados sob estereoscópio.

No estudo dos aspectos biológicos, ninfas móveis de $\boldsymbol{A}$. nerii, recém-eclodidas, foram transferidas, para as abóboras. Para a obtenção das ninfas recémeclodidas, as carapaças de inúmeras cochonilhas adultas foram levantadas, com auxílio de um estilete e estereoscópio. Com um pincel de cerdas finas, umedecidas com água destilada, foram transferidas 15 ninfas para cada abóbora, em área de $12 \mathrm{~cm}$ de diâmetro, demarcadas com cola "stick", segundo metodologia de TOMKINS et al. (1997).

Ciência Rural, v.36, n.2, mar-abr, 2006. 
Após a realização das transferências individuais das ninfas recém-eclodidas, as abóboras foram mantidas em câmaras climatizadas, sob temperatura de $25 \pm 1^{\circ} \mathrm{C}, 70 \pm 10 \%$ de UR e 12 horas de fotofase. A cada 60 minutos, foi observado se as cochonilhas já haviam se fixado à abóbora, até que todas as ninfas se fixassem, registrando assim, a duração da fase móvel do $1^{\circ}$ estádio ninfal.

Foi adotado o delineamento inteiramente casualizado, com 60 repetições (ninfas) para a fase ninfal. As ninfas foram observadas diariamente, para registrar a duração dos estádios e da fase ninfal, além da viabilidade.

Para estudar os aspectos reprodutivos e a longevidade de $\boldsymbol{A}$. nerii, foi necessária a demarcação de áreas circulares (3cm de diâmetro), com cola "stick", em volta das fêmeas. Para tanto, algumas fêmeas precisaram ser removidas, dando espaço para as que seriam avaliadas até a morte (36 fêmeas), registrandose a duração dos períodos pré-reprodutivo e reprodutivo, o número de ninfas produzidas e a longevidade. Diariamente, as ninfas produzidas eram contadas e removidas das áreas demarcadas, com auxílio de um estilete e estereoscópio.

\section{RESULTADOS E DISCUSSÃO}

Aspectos morfológicos e comportamentais de A. nerii

A linhagem unipariental de $\boldsymbol{A}$. nerii apresentou dois estádios ninfais, sendo o $1^{\circ}$ estádio composto por duas formas distintas, uma móvel e uma posterior, de maior duração, fixa. A fase de ninfa móvel apresenta coloração amarela, com presença de antenas e pernas, as quais possibilitam seu caminhamento, tão logo saem da carapaça materna. Alguns indivíduos tendem a se fixar ao redor da carapaça materna e de outras cochonilhas adultas, formando colônias.

No $1^{\circ}$ estádio, as ninfas fixam-se com a inserção dos estiletes no hospedeiro, neste caso a abóbora “cabotiá”. Após sua fixação, começam a produzir uma secreção cerosa sobre seu corpo, para impedir, provavelmente, o ressecamento e para garantir sua proteção. A quantidade de cera sobre a carapaça aumenta com o desenvolvimento da ninfa, tornandose mais espessa e rígida, formando um halo amarelo ao redor da primeira carapaça. Ao findar o $1^{\circ}$ estádio, a parte dorsal da exúvia é incorporada à face interna da carapaça.

No $2^{\circ}$ estádio, as ninfas ainda apresentam coloração amarelada. Dorsalmente, visualiza-se uma nova banda em torno da carapaça do $1^{\circ}$ estádio. A carapaça torna-se transparente ao seu redor, podendo observar o formato do corpo da ninfa, e, ao término desse estádio, a exúvia é incorporada da mesma forma descrita anteriormente, tornando a carapaça ainda mais rígida e maior. Nesse estádio a carapaça apresenta uma coloração pouco mais escura, voltando a sua cor amarelada no final do estádio com a contínua produção de cera até fim do desenvolvimento.

A fêmea permanece com sua cor amarelada, continua a secretar a cera para formação completa da carapaça, que se inicia a partir da última banda do $2^{\circ}$ estádio, expandindo-se ao redor e além do corpo da mesma. A carapaça, constituída por todas as bandas de secreção, toma formato circular e convexo, apresentando uma extremidade que permanece livre, constituindo-se na única passagem para as ninfas abandonarem a carapaça materna.

A reprodução é ovovivípara, sendo que as ninfas recém-eclodidas abandonam a carapaça materna à procura de um local adequado para a fixação, sendo de preferência locais como reentrâncias e rugosidades para sua proteção.

Aspectos biológicos de $\boldsymbol{A}$. nerii - Fase ninfal

A duração média da fase móvel do $1^{\circ}$ estádio foi de 2,4 $\pm 0,33$ horas (Tabela 1). ANDRADE (2005) observou que ninfas de Chrysomphalus aonidum (Linnaeus, 1758) se fixaram após $0,8 \pm 0,08$ horas, sobre o mesmo substrato e sob a mesma temperatura. Para Parlatoria cinerea Doane \& Hadden, 1909 foram necessárias 5,6 horas, a $24^{\circ} \mathrm{C}$, tendo laranjas como substrato (GRAVENA et al., 1993).

A fase fixa do $1^{\circ}$ estádio apresentou duração média de 8,3 $\pm 0,06$ dias (Tabela 1), diferindo dos resultados de TOMKINS et al. (1997), que observaram para esta espécie de diaspidídeo uma duração média de 13 dias, na mesma temperatura, porém tendo batata como substrato. Resultados diversos foram obtidos com outras espécies de diaspidídeos, como os 
Tabela 1 - Duração média e viabilidade dos estádios ninfais de Aspidiotus nerii ( $25 \pm 1^{\circ} \mathrm{C}$, UR de $70 \pm 10 \%$ e fotofase de 12 horas). Jaboticabal, SP. 2002/2003.

\begin{tabular}{lcccc}
\hline & \multirow{2}{*}{ Estádios } & \multicolumn{2}{c}{ Duração } & Viabilidade \\
\cline { 3 - 5 } & & Média \pm Erro padrão & Amplitude \\
\hline \multirow{2}{*}{$1^{\circ}$ estádio } & Fase móvel & $2,4 \pm 0,33$ horas & $1,8-3,0$ & $100 \%$ \\
$2^{0}$ estádio & Fase fixa & $8,3 \pm 0,06$ dias & $8,0-9,0$ & $100 \%$ \\
Fase ninfal & & $19,4 \pm 0,11$ dias & $18,0-21,0$ & $100 \%$ \\
\hline
\end{tabular}

registrados por GRAVENA et al. (1993), para $\boldsymbol{P}$. cinerea, na mesma temperatura, de 6,87 $\pm 1,17$ dias, em frutos de laranja; e por ANDRADE (2005), para C. aonidum, na mesma temperatura e substrato, de 5,5 dias.

O 2oestádio de $\boldsymbol{A}$. nerii apresentou duração

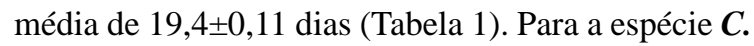
aonidum, ANDRADE (2005) observou duração média de 8,3 $\pm 0,07$ dias para esse estádio. Deve-se considerar que $\boldsymbol{C}$. aonidum apresenta três estádios ninfais, enquanto $\boldsymbol{A}$. nerii apresenta apenas dois. Com relação à fase ninfal, todas as ninfas avaliadas atingiram a fase adulta, correspondendo a 100\% de viabilidade(Tabela 1).

Aspectos biológicos de A. nerii - Fase adulta

A duração média do período préreprodutivo de $\boldsymbol{A}$. nerii foi de $17,3 \pm 0,18$ dias (Tabela 2 ). ANDRADE (2005) registrou período médio de 27,5 $\pm 0,53$ dias para $\boldsymbol{C}$. aonidum, sob a mesma temperatura e substrato de criação. $\boldsymbol{A}$. nerii apresentou um menor período pré-reprodutivo que $\boldsymbol{C}$. aonidum, favorecendo criações massais de coccinelídeos predadores de diaspidídeos.

A duração média do período reprodutivo foi de 68,2 $\pm 4,33$ dias (Tabela 2), sendo mais longo que de outros diaspidídeos. PARRA \& LOAYZA (2001)

Tabela 2 - Parâmetros reprodutivos médios e longevidade média de Aspidiotus nerii $\left(25 \pm 1^{\circ} \mathrm{C}\right.$, UR de $70 \pm 10 \%$ e fotofase de 12 horas). Jaboticabal, SP. 2002/2003.

\begin{tabular}{lcr}
\hline Parâmetros & \multicolumn{2}{c}{ Duração (dias) } \\
& Média \pm Erro padrão & Amplitude \\
\hline Período Pré-Reprodutivo & $17,3 \pm 0,18$ & $16,0-20,0$ \\
Período Reprodutivo & $68,2 \pm 4,33$ & $17,0-108,0$ \\
Longevidade & $100,5 \pm 4,51$ & $51,0-136,0$ \\
\hline
\end{tabular}

obtiveram período de 55,8 dias, a $25^{\circ} \mathrm{C}$, para Selenaspidus articulatus (Morgan, 1889). ANDRADE (2005) registrou período reprodutivo de 36,0 $\pm 5,65$ dias para C. aonidum, sob mesma temperatura.

O número médio de ninfas produzidas por fêmea foi $175,5 \pm 10,29$, representando $2,7 \pm 0,11$ ninfas/ fêmea/dia (Tabela 3). ANDRADE (2005) obteve 243,8 $\pm 40,06$ ninfas produzidas por fêmea de $\boldsymbol{C}$. aonidum, a $25^{\circ} \mathrm{C}$.

Com relação ao estudo da longevidade das fêmeas, observou-se média de 100,5 $\pm 4,51$ dias (Tabela 2). Estes dados são superiores aos registrados para $\boldsymbol{C}$. aonidum, $65,1 \pm 6,28$ dias, a $25^{\circ} \mathrm{C}$ (ANDRADE, 2005).

Aproximadamente $50 \%$ das ninfas são produzidas nos primeiros 30 dias de vida das fêmeas (Figura 1), sendo um fator favorável à criação massal para alimentar predadores, economizando tempo e espaço físico em laboratórios.

Em estudos de predação de $\boldsymbol{A}$. nerii pelos adultos do coccinelídeo $\boldsymbol{C}$. citricola, a $24^{\circ} \mathrm{C}$, SILVA et al. (2003) observaram predação de 4,38 $\pm 0,36$ ninfas por dia e um total médio de 46,38 ninfas durante o seu desenvolvimentolarval, sendo 51,2\% desse total no último estádio. Os adultos de $\boldsymbol{C}$. citricola predam, diariamente, mais ninfas do que adultos da cochonilha

Tabela 3 - Número médio de ninfas produzidas por fêmea de Aspidiotus nerii $\left(25 \pm 1^{\circ} \mathrm{C}\right.$, UR de $70 \pm 10 \%$ e fotofase de 12 horas). Jaboticabal, SP. 2002/2003.

\begin{tabular}{lcr}
\hline \multirow{2}{*}{ Parâmetros } & \multicolumn{2}{c}{ Número médio de ninfas } \\
& Média \pm Erro padrão & Amplitude \\
\hline Ninfas/Fêmea & $175,5 \pm 10,29$ & $59,0-266,0$ \\
Ninfas/Fêmea/Dia & $2,7 \pm 0,11$ & $1,6-4,1$ \\
\hline
\end{tabular}

Ciência Rural, v.36, n.2, mar-abr, 2006. 


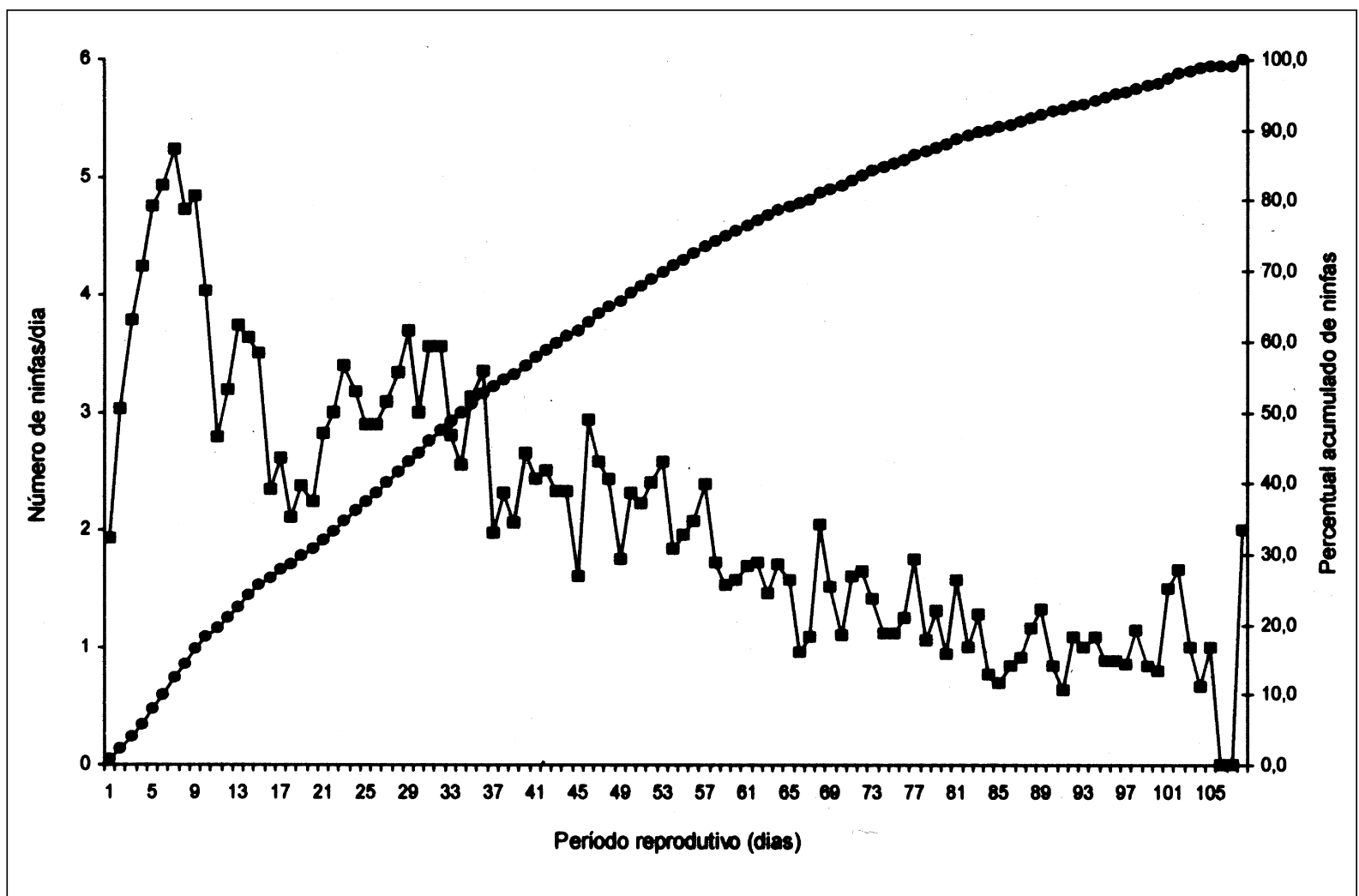

Figura 1 - Número de ninfas produzidas diariamente por fêmeas de Aspidiotus nerii e percentual acumulado de ninfas. Temperatura de $25 \pm 1^{\circ} \mathrm{C}$, UR de $70 \pm 10 \%$ e fotofase de 12 horas. Jaboticabal, SP. 2002/2003.

A. nerii. Os mesmos autores citam, ainda, altos valores relacionados à viabilidade de $\boldsymbol{C}$. citricola alimentado com $\boldsymbol{A}$. nerii, e observram também que a longevidade

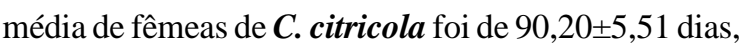
mostrando boa relação entre a longevidade da cochonilha e a do predador.

\section{CONCLUSÃO}

Aspidiotus nerii criada em laboratório sobre abóboras “cabotiá” apresenta excelente desenvolvimento, alta viabilidade ninfal, produz grande quantidade de ninfas por fêmea, favorecendo a sua utilização na criação massal de coccinelídeos predadores.

\section{AGRADECIMENTO}

À CAPES (Coordenação de Aperfeiçoamento de Pessoal de Nível Superior) pela bolsa de Mestrado concedida ao primeiro autor.

\section{REFERÊNCIAS}

ANDRADE, L.L. Aspectos morfológicos e bioecológicos de Chrysomphalus aonidum (Hemiptera: Diaspididae). 2005. 63f. Dissertação (Mestrado em Entomologia Agrícola) - Faculdade de Ciências Agrárias e Veterinárias, Universidade Estadual Paulista, Jaboticabal.

BEARDSLEY JÚNIOR, J.W.; GONZALEZ, R.H. The biology and ecology of armored scales. Annual Review of Entomology, Palo Alto, v.20, p.47-73, 1975.

BUSOLI, A.C. et al. Primeiro registro da ocorrência de Cybocephalus sp. (Coleoptera: Nitidulidae) predando Diaspididae (Hemiptera) em citros no Estado de São Paulo. In: SIMPÓSIO DE CONTROLE BIOLÓGICO, 9., 2005, Recife, PE. Anais... Recife: SEB, 2005. p.179.

DeBACH, P.; WHITE, E.B. Commercial mass culture of the California red scale parasite Aphytis lingnanensis. California Agricultural Experiment Station Bulletin, Berkeley, v.770, 58p, 1960.

FLANDERS, S.E. Mass culture of California red scale and its golden Chalcid parasites. Hilgardia, Oakland, v.20, p.1-42, 1951.

GARCIA, M.A. Ecologia nutricional de parasitóides e predadores terrestres. In: PANIZZI, A.R.; PARRA, J.R.P. Ecologia nutricional de insetos e suas implicações no manejo de pragas. Brasília: CNPq, 1991. p.289-312. 
GRAVENA. S. et al. Biologia de Parlatoria cinerea (Hemiptera: Diaspididae) e predação por Chrysoperla externa (Neuroptera: Chrysopidae). Científica, São Paulo, v.21, n.1, p.149-156, 1993.

GUERREIRO, J.C. Aspectos morfológicos e bioecológicos de Pentilia egena Mulsant, 1850 (Coleoptera: Coccinellidae). 2000. 94f. Dissertação (Mestrado em Entomologia Agrícola) - Faculdade de Ciências Agrárias e Veterinárias, Universidade Estadual Paulista, Jaboticabal.

NEUENSCHWANDER, P. et al. Biologie et écologie d'Aspidiotus nerii BOUCHE (Homoptera: Diaspididae) sur olivier en Crète Occidentale (Grèce). Fruits, Paris. v.32, n.6, p.418-421, 1977.

PARRA, J.R.P.; LOAYZA, R.M. Cochonilha-Pardinha, Selenaspidus articulatus (Hemiptera: Diaspididae). In: VILELA, E.F. et al. Histórico das pragas introduzidas no Brasil. Ribeirão Preto: Holos, 2001. p.53-57.

ROSE, M. Rearing and mass rearing. In: ROSEN, D. Armored scale insects their biology, natural enemies and control. Jerusalém: Elseiver, 1990. p.357-365.
SILVA, R.A. et al. Desenvolvimento e comportamento de predação de Coccidophilus citricola Brèthes, 1905 (Coleoptera: Coccinellidae) sobre Aspidiotus nerii Bouché, 1833 (Hemiptera: Diaspididae). Boletín de Sanidad Vegetal Plagas, Madrid, v.29, n.1, p.9-15, 2003.

SILVA, A.G.A. et al. Quarto catálogo dos insetos que vivem nas plantas do Brasil, seus parasitos e predadores. Rio de Janeiro: Ministério da Agricultura, 1968. T.1, 265p.

TASHIRO, H. Improved laboratory techniques for rearing California red scale on lemons. Journal of Economic Entomology, Lanhan, v.59, p.604-608, 1966.

TOMKINS, A.R. et al. Development of a model to predict the phenology of oleander scale (Aspidiotus nerii) infesting kiwifruit. Acta-Horticulturae, Wageningen, n.444, p.791795, 1997.

ZIMMERMAN, E.C. Insects of Hawaii: Homoptera: Sternoorhyncha. Honolulu: University of Hawaii, 1948. V.5, 464p. 